\title{
Low-Temperature Thermal Conductivity of Superconductors With Gap Nodes
}

\author{
Tomas Löfwander ${ }^{1,2}$ and Mikael Fogelström ${ }^{3}$ \\ ${ }^{1}$ Institut für Theoretische Festkörperphysik, Universität Karlsruhe, 76128 Karlsruhe, Germany* \\ ${ }^{2}$ Department of Physics \& Astronomy, Northwestern University, Evanston, Illinois 60208, USA \\ ${ }^{3}$ Applied Quantum Physics Laboratory, MC2, Chalmers University of Technology, S-412 96 Göteborg, Sweden
}

(Dated: March 16, 2005)

\begin{abstract}
We report a detailed analytic and numerical study of electronic thermal conductivity in $d$-wave superconductors. We compare theory of the cross over at low temperatures from $T$-dependence to $T^{3}$-dependence for increasing temperature with recent experiments on $\mathrm{YBa}_{2} \mathrm{Cu}_{3} \mathrm{O}_{7}$ in zero magnetic field for $T \in[0.04 K, 0.4 K]$ by Hill et al., Phys. Rev. Lett. 92, 027001 (2004). Transport theory, including impurity scattering and inelastic scattering within strong coupling superconductivity, can consistently fit the temperature dependence of the data in the lower half of the temperature regime. We discuss the conditions under which we expect power-law dependences over wide temperature intervals.
\end{abstract}

Low-temperature measurements of electronic transport properties have given a wealth of information about nodal quasiparticles deep in the superconducting phase of unconventional superconductors $1,2,2,3,4,5,6,7,8$, 9, 10, 11, 12, 13. The ultra-low temperature regime is of great interest because response functions such as the zero-frequency charge conductivity, thermal conductivity, and thermoelectric response function, are believed to be limited by elastic impurity scattering and obey power laws 14, 15, 16, 17]. Thermal conductivity is of particular importance in this context, since theory predicts universality in the sense that the low-temperature asymptotic does not depend on the properties of the impurity potential [18. For higher temperatures, of the order of the low-energy impurity band width $\gamma$ and higher, thermal conductivity is not universal and is sensitive to the details of impurity scattering [16].

The universal character of the $T \rightarrow 0$ thermal conductance was studied experimentally in great detail for several different cuprate materials for a wide range of doping levels [2, 4, [5, 6, 9, 10, 11]. Measurements of how the universal limit is approached as temperature is lowered was only recently reported by Hill et al 12 for $\mathrm{YBa}_{2} \mathrm{Cu}_{3} \mathrm{O}_{7}$. After subtracting a phonon contribution $\propto T^{3}$, they found an electronic contribution of the form $\kappa_{e l} / T=\left(\kappa_{0} / T\right)\left(1+k T^{2}\right)$, with $\kappa_{0} / T=0.16 \mathrm{~mW} /\left(\mathrm{K}^{2} \mathrm{~cm}\right)$ and the coefficient $k=19.2 K^{-2}$. This form is consistent with a Sommerfeld-type expansion of $\kappa_{e l}(T)$ (16] and below). However, the large value of $k$ implies an unrealistically clean sample if impurity scattering is in the unitary limit. On the other hand, for Born limit scattering, the concentration required to fit the large slope is unrealistically large and should lead to a severe reduction of the critical temperature which is not seen. Their plausible conclusion was therefore that scattering might be in between the unitary and Born limits.

In this paper we present results for thermal conductivity including effects of elastic impurity scattering and inelastic electron-boson scattering. We focus on how the universal limit is approached and make a detailed comparison with the experimental results of Hill et al 12]. Our results can be summarized as follows. (1) The main effect of inelastic scattering is an effective massdependence of the universal (with respect to impurity scattering) $T \rightarrow 0$ thermal conductance [19]. The coefficient $k$ is however effective mass independent. (2) We can fit the data 12] in the lower half of the $T$-interval with a best fit for a phase shift slightly below $90^{\circ}$. (3) The discrepancy at higher $T$ is related to the split of the low-energy impurity band, which makes it hard to observe power laws over wide $T$-intervals in ultra-clean materials.

The thermal conductivity $\kappa(T)$ of a superconductor has an electronic contribution $\kappa_{e l}(T)$, a phononic part $\kappa_{p h}(T)$, and possibly contributions from other existing excitations. We will only consider $\kappa_{e l}(T)$. To quantify the effects of inelastic scattering on the same footing as elastic impurity scattering we make a self-consistent strong-coupling calculation using quasi-classical Eliashberg theory 20] including impurity scattering in the $\hat{t}$-matrix approximation. We consider a two-spectra model for electron-boson interactions that generate both d-wave superconductivity and an inelastic scattering self energy. One spectrum gives coupling in the s-wave and $\mathrm{d}$-wave channels, but is attractive only in the d-wave. Hence, this part is responsible for pairing but also contributes to inelastic scattering. The second spectrum couples only in the s-wave channel (repulsively) and gives an incoherent background scattering. Even though the model is general we choose parameters that generates a $\mathrm{d}$-wave superconductor with properties close to that of a weakly coupled superconductor, with a spectroscopic gap $\Delta_{0}=2.59 T_{c}\left(T_{c}=0.13 \omega_{\text {mode }}\right)$ 21]. The temperature dependence $\kappa_{e l}(T)$ is displayed in Fig. 1 1 for several impurity concentrations $n_{\text {imp }}\left(\Gamma_{0}=n_{\text {imp }} / \pi \mathcal{N}_{f}\right)$ and near-resonant impurity scattering (phase shift $\delta_{0}=89.5^{\circ}$ ). We show in panels (a) and (b) the electron-boson coupling functions, $\chi^{2} I(\omega)$, and the resulting density of states, $N_{S}(\epsilon)$. The imaginary part of the inelastic self energy gives a $T$-dependent contribution to the scattering rate, $\Gamma_{\text {in }}(T)$ 


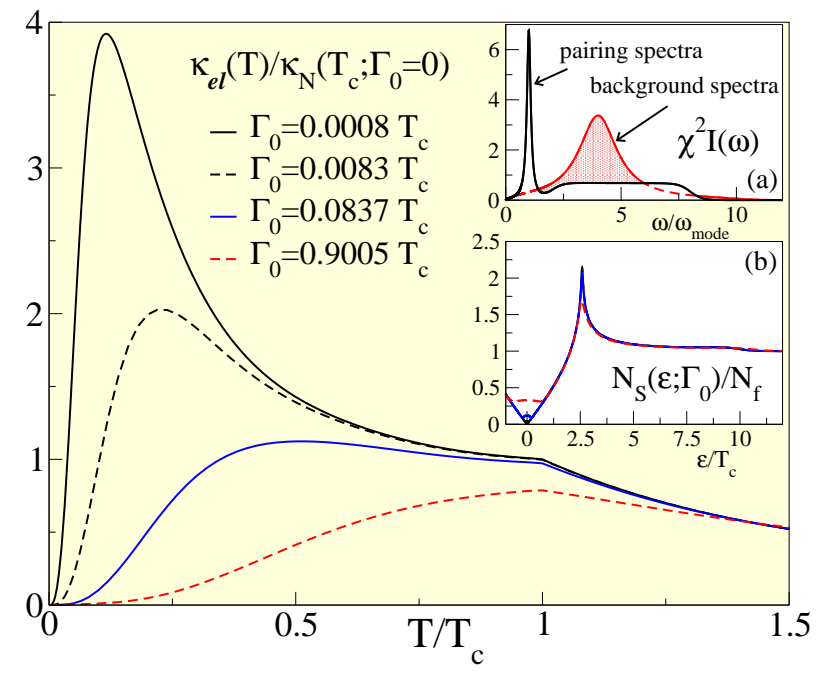

FIG. 1: Temperature dependence of the electronic contribution to the thermal conductivity for elastic scattering rates as indicated, for a phase shift $\delta_{0}=89.5^{\circ}$. Insert (a): the two electron-boson coupling spectra used in the calculations. Insert (b): single-particle density of states in the superconducting state at $T=0$.

22, 23, 24], while the contribution from elastic impurity scattering, $\Gamma=\Gamma_{0} \sin ^{2} \delta_{0}$, is $T$-independent. The relative importance of the two contributions leads to a crossover temperature $T^{*}$. Impurity scattering dominates in the low $-T$ region, while inelastic scattering dominates in the high $-T$ region. The cross-over is reflected in $\kappa_{e l}(T)$ as the peak at $T \sim T^{*}$ in Fig. 10 19, 24].

Although the imaginary part of the inelastic selfenergy $\hat{\Sigma}_{\text {in }}^{R}$ is small for $T \ll T^{*}$, the real part remains important. By using general symmetry relations we can write down the renormalization of the energy from both elastic and inelastic scattering as

$$
\tilde{\epsilon}^{R}=\epsilon-\frac{1}{2} \operatorname{Tr}\left[\hat{\tau}_{3} \hat{\Sigma}^{R}(\epsilon)\right]=i \gamma+(1+\lambda) \epsilon+\mathcal{O}\left[\epsilon^{2}\right] .
$$

Here is $\gamma$ solely due to impurity scattering. The slope of the real part of $\tilde{\epsilon}^{R}(\epsilon)$ has contributions from both impurity scattering and inelastic scattering, $\lambda=\lambda_{\text {imp }}+\lambda_{\text {in }}$. Because $\Re \Sigma_{\text {in }}^{R}(\epsilon)$ is linear in energy up to the modeenergy, $\omega_{\text {mode }}>\Delta_{0} \gg T^{*}$, an effective theory can be made for $T \ll T^{*}$ by dividing the transport equation by $1+\lambda_{\text {in }}$ evaluated in the superconducting phase. The inelastic part is then interpreted as an effective mass via $m^{*} / m=1+\lambda_{\text {in }}$. The contribution from impurity scattering on the other hand, has a non-trivial energy dependence for $\epsilon \lesssim \Delta_{0}$, and can not be divided out. Within the effective theory, we use Eq. (11) and the method of Graf et al [16] to write down the low $-T$ form

$$
\frac{\kappa_{e l}(T)}{T}=\frac{\pi^{2}}{3} \frac{2 \mathcal{N}_{f} v_{f}^{2}}{\pi \mu \Delta_{0}}\left[1+\frac{7 \pi^{2}}{15} \frac{\tilde{a}^{2} T^{2}}{\gamma^{2}}\right]+\mathcal{O}\left[\left(\frac{T}{\gamma}\right)^{4}\right] \text {. }
$$

Here are $\mathcal{N}_{f}$ and $v_{f}$ the effective density of states at the Fermi level and the effective Fermi velocity, while
$\Delta_{0}$ is the spectroscopic gap and $\mu$ is the opening rate of the gap function at the node. We now find that the remaining effect at $T \ll T^{*}$ of inelastic scattering within strong-coupling superconductivity is a modification of the $T \rightarrow 0$ asymptotic of the thermal conductance. When we write explicitly, $\mathcal{N}_{f} \rightarrow \mathcal{N}_{f}^{*}=\mathcal{N}_{f}^{0}\left(1+\lambda_{\text {in }}\right)$, and $v_{f} \rightarrow v_{f}^{*}=v_{f}^{0} /\left(1+\lambda_{\text {in }}\right)$, one factor $1+\lambda_{\text {in }}$ remains in the denominator. Within the bare theory, this result can be traced back to that the spectroscopic gap $\Delta_{0}$ in the weak-coupling limit is replaced by the strong coupling off-diagonal function $W(0)$ [19]. In our model $W(\epsilon)$ is a weak function of energy and to a good approximation $W(0) \approx \Delta_{0}\left(1+\lambda_{\text {in }}\right)$. This effective mass dependence is in sharp contrast to the normal state, in which case $\kappa_{e l}^{N}(T)=T\left(\pi^{2} / 3\right) \mathcal{N}_{f} v_{f}^{2} / 2 \Gamma$ is independent of $m^{*}$, because of canceling factors of $\left(1+\lambda_{\text {in }}\right)$ in $N_{f}, v_{f}^{2}$ and $\Gamma$ in accordance with general strong-coupling results for transport coefficients [25]. In this sense, transport properties of quasiparticles in the low-energy nodal impurity band is quite different from quasiparticles in the normal state.

Effective-mass factors from $\tilde{a}$ and $\gamma$ cancel in the coefficient $k=\frac{7 \pi^{2}}{15}(\tilde{a} / \gamma)^{2}$ in Eq. (2) and it is only dependent on properties of the impurity potential. In addition to the electron-hole symmetric part above, also the electronhole asymmetric part of the impurity self-energy enters [26]. Only the imaginary part

$$
\frac{1}{2} \operatorname{Tr}\left[\Im \hat{\Sigma}_{\text {imp }}^{R}(\epsilon)\right]=c \epsilon+\mathcal{O}\left[\epsilon^{3}\right] .
$$

contributes to the conductivity through $\tilde{a}^{2}=\left(1+\lambda_{\text {imp }}\right)^{2}+$ $2 c^{2} \quad$ 17. The presence and importance of $c$ represents the fact that electron-like and hole-like quasiparticles have different transport lifetimes above and below the Fermi level, except in the strict unitary and Born limits for which it vanishes.

We now discuss the experiment [12] in terms of the presented effective theory and its low $-T$ expansion in Eq. (2) with the parameters $\left\{\gamma, \lambda_{\text {imp }}, c\right\}$ computed selfconsistently [17]. Starting for simplicity with $s$-wave impurity scattering, we present in Fig. 2(d) several combinations of impurity concentrations and phase shifts that give good fits to the data in the lower half of the $T$-interval. In fact, there is a broad range in parameter space for which the slope $k=19.2 K^{-2}$ can be reproduced [blue dash-dotted lines in Fig. 2(a)-(c)]. Similarly, we can find many combinations yielding other values of $k$ (black and red lines). However, not all combinations of parameters produce a consistent fit, in the sense that the expansion Eq. (2) represents over a wide temperature range the full numerically computed thermal conductivity shown in Fig. 2(d). The deviation from true $T^{2}$-behavior is also seen in the figure: the three curves start at low $T$ with the same coefficient, $k=19.2 K^{-2}$, but deviate at higher $T$. This is also the reason for the discrepancy between theory and experiments in the upper half of the $T$-interval. The part in parameter space 

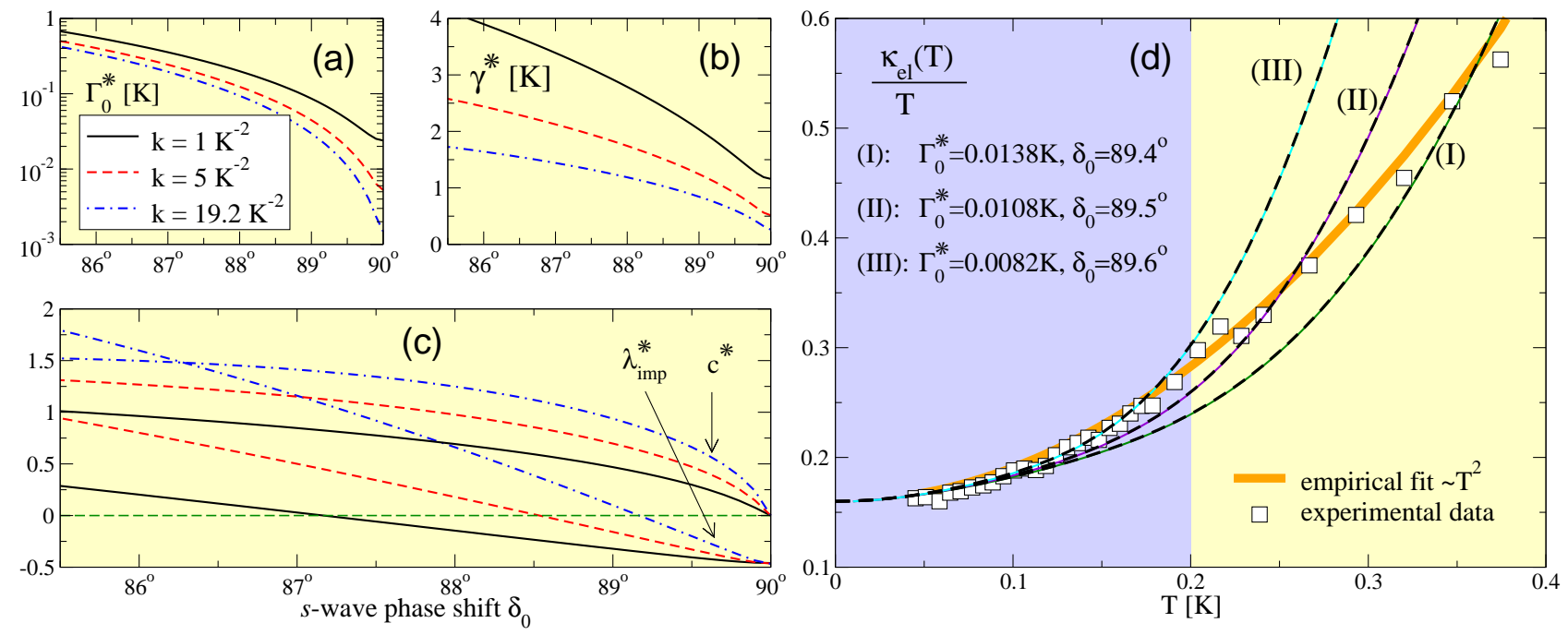

FIG. 2: (a)-(c) The low-temperature parameters in Eqs. (1) and 3) for three values of the $T^{2}$-coefficient in Eq. 22 as function of scattering phase shift. (d) Three sets of parameters that represents our best fits to the data of Ref. 12]. The blue shaded region indicates the temperature range where a good fit can be made. The dashed curves are from a strong-coupling calculation while the underlying solid lines are generated by the effective theory. Curve (II) is the low $-T$ part of the black solid line in Fig. 1 In all cases $k=19.2 K^{-2}$. Note that the material appears cleaner in the effective theory as $\Gamma_{0}^{*}=\Gamma_{0} /\left(1+\lambda_{\text {in }}\right)$ with $\Gamma_{0}=n_{\text {imp }} / \pi \mathcal{N}_{f}$. We normalized $\kappa_{e l}(T) / T$ to the $T \rightarrow 0$ extrapolated value $0.16 \mathrm{~mW} /\left(\mathrm{K}^{2} \mathrm{~cm}\right)$. The energy scale is $T_{c}=90 \mathrm{~K}$.

for which the Sommerfeld expansion is applicable can be broadly defined as phase shifts for which $\lambda_{\text {imp }}$ is negative.

Let us discuss in more detail why Eq (2) works well only in a restricted low- $T$ interval. Recall that the thermal conductivity can be written as $\kappa_{e l}(T)=$ $\int_{-\infty}^{\infty} d \epsilon K(\epsilon)\left(-\frac{\partial f}{\partial \epsilon}\right)$, where $f$ is the Fermi function. The derivative of the Fermi function is sharply peaked at the Fermi level in an interval of order $T$, which leads us to expand all quantities around $\epsilon=0$, as in Eqs. (11) and (3), and do a Sommerfeld expansion $\kappa_{e l}(T)=K(0)+$ $\left.\sum_{n=1}^{\infty} \frac{d^{2 n} K(\epsilon)}{d \epsilon^{2 n}}\right|_{\epsilon=0} a_{n} T^{2 n}$, where the for us relevant coefficients $a_{1}=\pi^{2} / 6$ and $a_{2}=7 \pi^{4} / 360$. However this scheme works well only if the function $K(\epsilon)$ is slowly varying on the scale $T$. In our case we can write $K(\epsilon)=\epsilon^{2} \tilde{\kappa}(\epsilon)$ and study the energy dependence of $\tilde{\kappa}(\epsilon)$, see Fig. 3(d). The fine structure at low energies for phase shifts below $90^{\circ}$, can intuitively be understood by studying the phase shift dependence of the self-energy. In a $d$-wave superconductor with a single impurity scattering strongly (near the unitary limit) there is a resonance in the $\hat{t}$-matrix at 27] $\epsilon_{\text {res }} \approx \pm \pi \cot \delta_{0} /\left[2 \ln \left(8 / \pi \cot \delta_{0}\right)\right]$ where the sign corresponds to the electron and hole sectors. The width of the resonance is smaller than the resonance energy by a factor $1 / \ln \left(8 / \pi \cot \delta_{0}\right) \ll 1$. Within transport theory we employ an impurity averaging technique [28] to obtain the self-energy for a material with a small concentration of randomly distributed impurities. As a result of the configuration average, the single impurity resonance is broadened into an impurity band of width $\gamma_{b}$, centered around an energy slightly shifted from the single impurity resonance $\epsilon_{\text {res }} \rightarrow \epsilon_{b}$. The impurity bands are shown in Fig. 3(a)-(c). For clean systems with a phase shift deviating substantially from $90^{\circ}$, the resulting bands are split into two parts symmetrically positioned above and below the Fermi level. The critical point is when the slope at zero energy of the real part of the self energy changes sign, i.e. when $\lambda_{\text {imp }}=-\Re \Sigma_{3}^{R}(0)$ turns positive. The structures in the self-energy lead to structures also in the response functions at low temperatures on the scale $T \sim \epsilon_{b} \ll \gamma_{b}$, see Fig. B](d), and the applicability of Eq. (2) is drastically reduced in temperature by the new constraint $T \ll \epsilon_{b}$.

In a more realistic model, impurity scattering is anisotropic. The main technical complication in this case is the necessity to take care of vertex corrections. They are small in the limit $T \ll \gamma[18$, but are important for higher temperatures. In particular they contribute to the coefficient $\tilde{a}$ in Eq. (2). For definiteness we have included $p$-wave and $d$-wave scattering channels and considered arbitrary phase shifts [29]. In general we obtain a pair of resonances, one electron-like and one hole-like (symmetrically positioned on each side of the Fermi level), for each phase shift. This fact was also noted recently in Ref. [30]. The main conclusion relevant in the present discussion is that the impurity self-energy aquires more structures (related to the new resonances) and the range of applicability of Eq. (2) is not improved.

In summary, let us list our results and make a few remarks. (1) Inelastic scattering contributes to the $T \rightarrow 0$ asymptotic (i.e. $\kappa_{0} \propto T$ ), but plays no role for the $T^{3}$ coefficient (i.e. $k$ ). (2) The low $-T$ half of the experimental data can be consistently fitted with parameters representing a wide range of impurity concentra- 

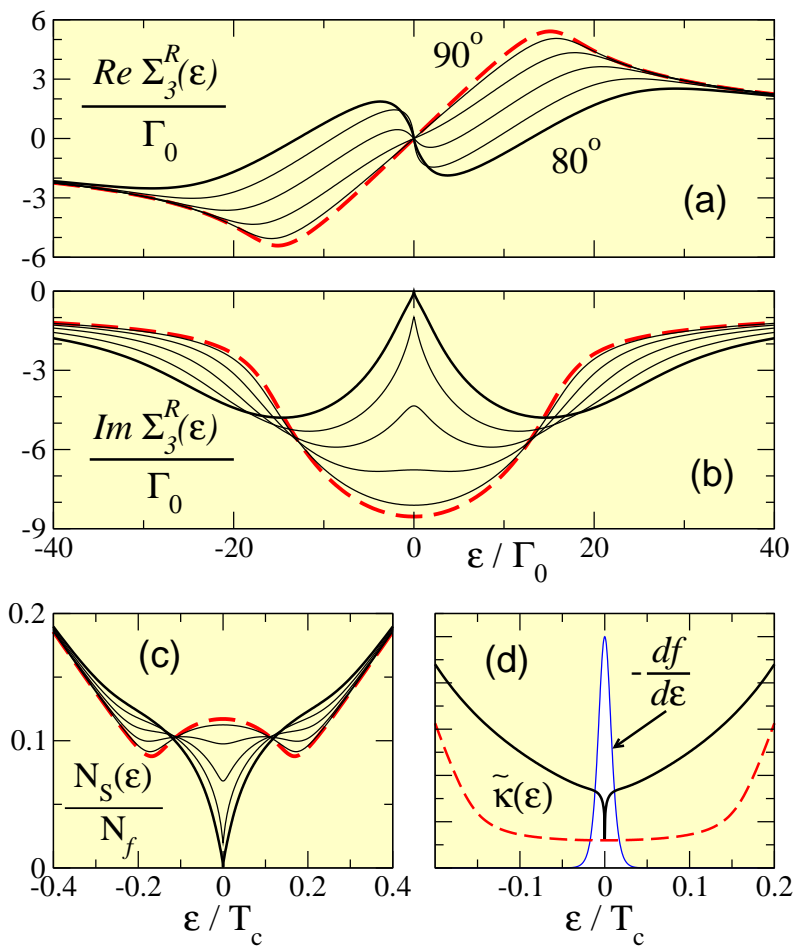

FIG. 3: (a)-(b) The impurity scattering self-energy and (c) the density of states for $\Gamma_{0}=0.01 T_{c}$ and phase shifts ranging from $90^{\circ}$ (red dashed line) to $80^{\circ}$ (thick black line) in steps of two degrees. (d) The kernel $\tilde{\kappa}(\epsilon)$ of the thermal conductivity contains structures at low energies for phase shifts below $90^{\circ}$ because of the split impurity bands seen in (a)-(c). Also shown is the derivative of the Fermi function which has a peak at the Fermi level of width $\sim T$. Here is $T=0.005 T_{c}=0.45 K$ for $T_{c}=90 K$.

tions and phase shifts. The best fit to the large coefficient $k=19.2 K^{-2}$ is obtained for a rather clean material with a scattering phase shift slightly below the unitary limit $\left(\delta_{0}<90^{\circ}\right)$. (3) However, we find deviations in the high $-T$ region. The reason is that the expansion Eq. (2) has a limited range of applicability, smaller than the temperature range in which the $T^{3}$ term was found experimentally to dominate. Indeed, in an earlier discussion of the data [12] in Ref. 31], model parameters were chosen so that the expansion Eq. (2) was inapplicable. In their work the unit-term Eq. (3) was neglected, which changed their results by about a factor two. We conclude that a clear cross over between $T$-dependence to $T^{3}$-dependence, with the respective power laws holding in wide $T$-intervals, is most easily observable in dirty to intermediately clean, but not in ultra-clean, materials. Finally, an alternative way of extracting information about impurity scattering from transport properties, would be to study the thermoelectric effect [17. In this case, the non-universality of the $T \rightarrow 0$ asymptotic can be used. This quantity, the analogue of $\kappa_{0}$, should be easier to measure and interpret than the $T^{3}$-part of $\kappa_{e l}$.

We gratefully acknowledge financial support from the Swedish Research Council (M.F.), the Wenner-Gren foundations (T.L.), and the EC under the Spintronics Network RTN2-2001-00440 (T.L.).

* Present address.

[1] D. A. Bonn, P. Dosanjh, R. Liang, and W. N. Hardy, Phys. Rev. Lett. 68, 2390 (1992).

[2] L. Taillefer, B. Lussier, R. Gagnon, K. Behnia, and H. Aubin, Phys. Rev. Lett. 79, 483 (1997).

[3] J. Corson, J. Orenstein, S. Oh, J. O'Donnell, and J. N. Eckstein, Phys. Rev. Lett. 85, 2569 (2000).

[4] M. Chiao et al., Phys. Rev. B 62, 3554 (2000).

[5] S. Nakamae et al., Phys. Rev. B 63, 184509 (2001).

[6] J. Takeya, Y. Ando, S. Komiya, and X. F. Sun, Phys. Rev. Lett. 88, 077001 (2002).

[7] C. Proust, E. Boaknin, R. W. Hill, L. Taillefer, and A. P. Mackenzie, Phys. Rev. Lett. 89, 147003 (2002).

[8] Y. Ando, J. Takeya, Y. Abe, X. F. Sun, and A. N. Lavrov, Phys. Rev. Lett. 88, 147004 (2002).

[9] M. Suzuki et al., Phys. Rev. Lett. 88, 227004 (2002).

[10] M. Sutherland et al., Phys. Rev. B 67, 174520 (2003).

[11] R. Bel et al., Phys. Rev. Lett. 92, 177003 (2004).

[12] R. W. Hill et al., Phys. Rev. Lett. 92, 027001 (2004).

[13] X. F. Sun, K. Segawa, and Y. Ando, Phys. Rev. Lett. 93, 107001 (2004).

[14] P. A. Lee, Phys. Rev. Lett. 71, 1887 (1993).

[15] Y. Sun and K. Maki, Europhys. Lett. 32, 355 (1995).

[16] M. J. Graf, S.-K. Yip, J. A. Sauls, and D. Rainer, Phys. Rev. B 53, 15147 (1996).

[17] T. Löfwander and M. Fogelström, Phys. Rev. B 70, 024515 (2004).

[18] A. C. Durst and P. A. Lee, Phys. Rev. B 62, 1270 (2000).

[19] E. Schachinger and J. P. Carbotte, Phys. Rev. B 57, 13773 (1998).

[20] D. Rainer and J. A. Sauls, in Superconductivity: From Basic Principles to the Latest Developements, edited by L. N. Butcher and Y. Lu (World Scientific, Singapore, 1995), pp. $45-78$.

[21] The electron-boson coupling strength is $\lambda_{\text {in }}=6.0$, with $48.5 \%$ contribution from $\lambda_{\mathrm{d} \text {-wave }}$ and $51.5 \%$ from $\lambda_{\text {incoh }}$.

[22] A. J. Millis, S. Sachdev, and C. M. Varma, Phys. Rev. B 37, 4975 (1988).

[23] P. J. Hirschfeld, W. O. Putikka, and D. J. Scalapino, Phys. Rev. Lett. 71, 3705 (1993).

[24] P. J. Hirschfeld and W. O. Putikka, Phys. Rev. Lett. 77, 3909 (1996).

[25] R. E. Prange and L. P. Kadanoff, Phys. Rev. 134, A566 (1964).

[26] H. Monien, K. Scharnberg, and D. Walker, Solid State Commun. 63, 263 (1987).

[27] A. V. Balatsky, M. I. Salkola, and A. Rosengren, Phys. Rev. B 51, 15547 (1995).

[28] A. A. Abrikosov, L. P. Gorkov, and I. E. Dzyaloshinski, Methods of Quantum Field Theory in Statistical Physics (Dover Publications, Inc., New York, 1975).

[29] The details of this calculaton will be presented elsewhere.

[30] T. S. Nunner and P. J. Hirschfeld, cond-mat/0411321.

[31] W. Kim, F. Marsiglio, and J. P. Carbotte, Phys. Rev. B 68, 174513 (2003). 